\title{
Does bisphosphonate treatment reduce the risk of future cancer?
}

\author{
Michael Pazianas ${ }^{1}$, Bo Abrahamsen ${ }^{2,3}$ \\ ${ }^{1}$ Institute of Musculoskeletal Sciences, Oxford University, Oxford, UK; ${ }^{2}$ Department of Medicine, Holbaek Hospital, Holbaek, Denmark; ${ }^{3}$ Odense \\ Patient Data Explorative Network, Institute of Clinical Research University of Southern Denmark and Odense University Hospital, Odense C, \\ Denmark \\ Correspondence to: Michael Pazianas, MD. Institute of Musculoskeletal Sciences, Oxford University, Oxford OX3 7LD, UK. \\ Email: Michael.Pazianas@ndorms.ox.ac.uk. \\ Comment on: Tao MH, Chen S, Freudenheim JL, et al. Oral bisphosphonate use and lung cancer incidence among postmenopausal women. Ann \\ Oncol 2018;29:1476-85.
}

Submitted Oct 13, 2018. Accepted for publication Oct 15, 2018.

doi: $10.21037 /$ cco.2018.10.08

View this article at: http://dx.doi.org/10.21037/cco.2018.10.08

Bisphosphonates are the first line of treatment for osteoporosis and metastatic bone cancer. It is almost fifty years since their antiresorptive properties were described and subsequently were used clinically. They were first used in a child with fibrodysplasia ossificans progressiva (FOP) [1969], then in four patients with active Paget's disease of bone [1971], and in the early 1980s in various types of cancer (1). However, the large scale use of bisphosphonates in the USA started in the second half of the 1990s, when the dual-energy X-ray absorptiometry (DXA) technology provided the tool for reproducible measurement of bone mineral density (BMD) and the nitrogen-containing or second generation bisphosphonates were introduced in clinical practice for the treatment of osteoporosis. Originally, with the approval of Alendronate (oral), followed by Risedronate (oral), then Ibandronate (oral and IV), and finally Zoledronate (IV) in 2007. The bisphosphonates enjoyed a steady rise in use up to 2008 followed by a constant and substantial fall since then (2) (Figure 1), despite their effectiveness and consensus that their benefits outweigh their known risks (3).

Interestingly, there have been claims that the beneficial effects of nitrogen-containing bisphosphonates could be extended beyond the skeletal system. Specifically, patients treated with nitrogen-containing bisphosphonates could be at lower risk of developing malignancies (4).

In a recently published paper, Tao et al., using data from the Women's Health Initiative (WHI) study, investigated the effects of bisphosphonate use on the incidence of lung cancer (5). They included 151,432 postmenopausal women, age 50 to 79 years old at baseline and anticipated survival of at least 3 years. The recruitment from 40 clinical centers across the United States took place between 1993 and 1998 and observations were censored at the last follow-up or 30 September 2013. As it was expected, the oral bisphosphonate use at baseline was only $2.1 \%$ with a modest increase to $9.7 \%$ by year 6 . More than $90 \%$ of users were on Alendronate. While the crude incidence of lung cancer in those who reported any type of bisphosphonate use was similar with the nonusers, there was a strong interaction with smoking. Hence, when the data were analyzed according to participants' smoking history, oral bisphosphonates were inversely associated with lung cancer risk only in never smoking women ( $\mathrm{HR}=0.57,95 \%$ CI, 0.39-0.84, $\mathrm{P}<0.01)$ and not in ever smokers. The association was stronger for total duration of use $\geq 1.5$ years ( $\mathrm{HR}=0.36,95 \% \mathrm{CI}, 0.18-0.73$ ).

There have been attempts to address a similar question in the past. Two UK retrospective studies reported a nonsignificant inverse association or no association $(6,7)$. Also, two retrospective studies using Taiwan health insurance system database reported contradicting findings, i.e., a positive association and no association respectively $(8,9)$. However, none of the above studies collected comprehensive information on risk factors of lung cancer, including smoking. Though the analysis was adjusted for smoking, this does not rule out an interaction with smoking such as reported here.

One explanation for these contradictory outcomes could be that the effects of bisphosphonate use on the incidence of different types of cancer were not a predetermined study outcome, especially in the WHI 


\section{Page 2 of 4}

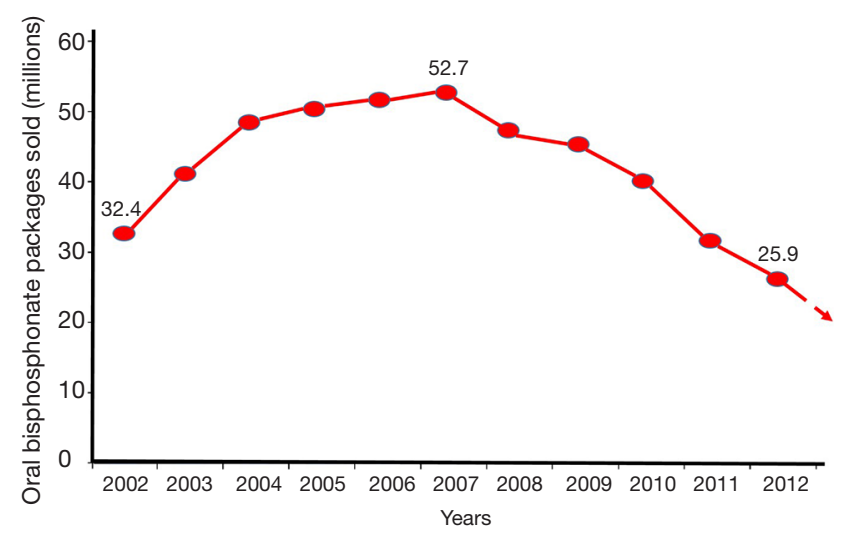

Figure 1 Oral bisphosphonate packages (alendronate, risedronate, and ibandronate) sold in the USA. Adapted with permission from Bone (2).

study conceived in the late ' 80 s to early ' 90 s, and the first FDA approval for a bisphosphonate (Alendronate) being granted in the second part of the 90's. Also differences in the design and analytical approach of these studies could have played a role. Observational cohorts have indeed reported mutually conflicting findings regarding different types of malignancies such as gastrointestinal cancer, in bisphosphonate users (10).

Very recently, new randomized controlled trial (RCT) data became available which suggest that even very low frequency dosing of a bisphosphonate with strong affinity to the skeleton and a short plasma half-life can significantly cut overall cancer risk and mortality. In a study of 2,000 postmenopausal women with osteopenia, i.e., modestly reduced $\mathrm{BMD}$, treated with zoledronic acid at 18 -month intervals for up to 6 years, the odds ratio for malignant diseases overall was 0.67 (95\% CI, 0.50-0.89) and the risk of all-cause mortality nominally but not significantly reduced by a third (11).

There are biologically plausible mechanisms by which nitrogen-containing bisphosphonates may reduce cancer risk. They exert their effects through the mevalonate pathway, the same pathway targeted by the cholesterollowering drugs statins, albeit downstream. As a result, they interfere with intracellular signaling pathways which are involved in cancer development. They inhibit prenylation in cancer cells, including human lung cancer cells (12). Furthermore, among others, in colorectal cancer, local administration of bisphosphonates in animals with chemically-induced ulcerative colitis and local exposure to a procarcinogen, ameliorates inflammation and significantly reduces the development of tumors (4).

Nitrogen-containing bisphosphonates exert antiangiogenic effects through the mevalonate pathway and also by inhibiting the expression of vascular endothelial growth factor (VEGF), a potent angiogenic factor (13). In human non-small-cell lung cancer cell line zoledronate induced dose-dependent reduction both of mRNA and protein expression of VEGF associated with parallel decrease in VEGF secretion in the culture medium (14). Clinically, administration of zoledronate, lowers significantly serum VEGF concentrations, although only modestly (non-significant) in those treated with less potent oral preparations, such as alendronate (15).

Overexpressed or mutated members of the human epidermal growth factor receptor (EGFR) family drive a number of common malignancies, including lung, breast and colorectal cancer. Nitrogen-containing bisphosphonates interact with the EGFR, a tyrosine kinase receptor, to cause apoptosis and reduce tumor volume (16). In addition, nitrogen-containing bisphosphonates inhibit tumor growth through activation of $\delta \delta$ T-cell receptor (17).

Bisphosphonates could also act indirectly through macrophages, which are osteoclast precursors. Macrophages are central in the growth and migration of tumor cells and in the production of tumor infiltrating macrophages. When activated, they may produce enhanced amounts of inflammatory mediators and growth factors and thereby increase the risk of cancer and its progression (4). Like osteoclasts, macrophages are highly endocytic cells capable of internalizing bisphosphonates; the endocytic capacity of other types of cells such as breast cancer cells is significantly less (18).

The effects of nitrogen-containing bisphosphonates are systemic and when administered IV (once a year or less frequently than that for the treatment of osteoporosis) they could achieve briefly high concentrations in the circulation albeit their high affinity to hydroxyapatite crystals results in their rapid removal from the circulation, almost exclusively through the kidneys, unmetabolized. Oral formulations however, usually administered weekly in osteoporotic patients, could have local effects on the colorectal mucosa, as well. This is because they are poorly absorbed (less than $1 \%$ ). The remaining more than $99 \%$ travels through the digestive tract. In the colorectal segment, its mucosa will come in contact with the bisphosphonate as it slowly moves through and in high concentrations well within the millimolar range.

Another significant factor we should take in account when we try to assess the anticipated effects of real-world 
bisphosphonate treatment on organs other than the skeleton, is their bone selectivity complimented by a notoriously suboptimal adherence. It has been estimated that one third to one half of patients do not take their medication as directed (compliance), and nonadherence may begin soon after treatment initiation (19). Even in countries reporting relatively good persistence (how long a patient continues therapy) with osteoporosis treatment, the mean persistence on oral bisphosphonates is only about 3 years (20). In addition, with oral bisphosphonates, even if they are taken correctly in terms of dose and frequency, when taken with food or beverages create complexes and they cannot be absorbed. This is why the routine requiring overnight fasting prior to taking the medication, continuing for up to 2 hours afterwards is mandatory but at the same time it has been one of the main reasons for poor adherence.

Based on the pharmacokinetics of the nitrogencontaining bisphosphonates, especially those administered orally, it is expected that the most noticeable effects on the cancer incidence, should be on the large intestine, where both circulating and locally applied bisphosphonates could exert maximum effects. If the effect is questionable, then, reported beneficial effects on other organs would be less convincing. Especially a reported decrease of the incidence of lung cancer after only two weeks of oral alendronate, the less potent nitrogen-containing bisphosphonate used for the treatment of osteoporosis, despite its strong statistical significance (5).

The IV use of nitrogen-containing bisphosphonates (almost exclusively Zoledronate), although it would achieve briefly high circulating concentrations, because they are administered in yearly long intervals, it may not be enough to sustain the level of presence required to achieve any clinically evident effect. Of course, from the embedded already in the bone bisphosphonates there will be a measurable amount released during resorption taking place in a new site, but this, to a great extent, will be taken up rapidly by the skeleton.

Another explanation for the apparent lack of anti-cancer effects in observational studies may be the duration of use, which may be more critical for non-skeletal outcomes than for bone outcomes. Given the established effects of the nitrogen-containing bisphosphonates on several cancer cell lines, their interaction with the EGFR (21) and the low endocytic capacity of cancer cells, it is most likely that the doses should be higher than those used for osteoporosis. Obviously, increasing the dose of currently approved nitrogen-containing bisphosphonates is not advisable. The alternative could be the administration of bisphosphonates with very low bone affinity. Even then, however, monocytes/ macrophages will be far ahead in the queue to phagocytose them and thus become neutralized, leading to depletion of these vital components of the innate immunity and this is not advisable either.

Hopes also have been raised with the use of statins, which like the nitrogen-containing bisphosphonates, target the mevalonate pathway. Currently, there is no compelling evidence that statins can prevent the onset of cancer (22). There is no evidence that patients on both medications, i.e., bisphosphonates and statins have lower cancer risk. Therefore, there is no indication to be used as chemoprotective agents.

The use of nitrogen-containing bisphosphonates in clinical therapeutics is likely to continue well into the future. Still it is difficult to imagine that their use will achieve, let alone surpass, the 2007 levels and see adherence improve. Their reputation has been damaged rather irreversibly because the strong dose of alarmism from the popular media on the rare adverse effects of osteonecrosis of the jaws and atypical femoral fractures, went unanswered for so long. At the same time, the prescription pattern has changed. A more sparing use and extensive use of drug holiday is now an established trend. The duration of treatment has become more personalized and the number of patients on bisphosphonates for more than five years has been reduced. The other drawback affecting initiation of treatment in the first place and then in the adherence is that osteoporosis is a silent disease without symptoms, even in cases of vertebral fractures and the perception that it is not a deadly disease, like heart disease for example. Therefore, the most likely scenario currently is that the use of bisphosphonates, in the broad term, it will be rather sporadic and probably not long enough to achieve long lasting effects on the risk of cancer unless employed specifically for that purpose. However, the potential, when administered intravenously, to achieve substantial cancer risk reductions even with doses spaced many months apart is certainly attractive even if hard to reconcile with the pharmacokinetics of bisphosphonates.

\section{Acknowledgments}

None.

\section{Footnote}

Conflicts of Interest: B Abrahamsen receives institutional 
research contract funds from UCB and Novartis. M Pazianas has no conflicts of interest to declare.

\section{References}

1. Russell RG. Bisphosphonates: the first 40 years. Bone 2011;49:2-19.

2. Wysowski DK, Greene P. Trends in osteoporosis treatment with oral and intravenous bisphosphonates in the United States, 2002-2012. Bone 2013;57:423-8.

3. Pazianas M, Abrahamsen B. Safety of bisphosphonates. Bone 2011;49:103-10.

4. Pazianas M, Russell RG. Potential therapeutic effects of oral bisphosphonates on the intestine. Ann N Y Acad Sci 2011;1240:E19-25.

5. Tao MH, Chen S, Freudenheim JL, et al. Oral bisphosphonate use and lung cancer incidence among postmenopausal women. Ann Oncol 2018;29:1476-85.

6. Cardwell CR, Abnet CC, Veal P, et al. Exposure to oral bisphosphonates and risk of cancer. Int J Cancer 2012;131:E717-E725.

7. Vinogradova Y, Coupland C, Hippisley-Cox J. Exposure to bisphosphonates and risk of common non-gastrointestinal cancers: series of nested case-control studies using two primary-care databases. Br J Cancer 2013;109:795-806.

8. Lee WY, Sun LM, Lin MC, et al. A higher dosage of oral alendronate will increase the subsequent cancer risk of osteoporosis patients in Taiwan: a population-based cohort study. PLoS One 2012;7:e53032.

9. Chiang $\mathrm{CH}$, Huang $\mathrm{CC}$, Chan WL, et al. Oral alendronate use and risk of cancer in postmenopausal women with osteoporosis: a nationwide study. J Bone Miner Res 2012;27:1951-58.

10. Passarelli MN, Newcomb PA, LaCroix AZ, et al. Oral bisphosphonate use and colorectal cancer incidence in the Women's Health Initiative. J Bone Miner Res 2013;28:2043-8.

11. Reid IR, Horne AM, Mihov B, et al. Fracture Prevention with Zoledronate in Older Women with Osteopenia. N Engl J Med 2018;379:2407-16.

Cite this article as: Pazianas M, Abrahamsen B. Does bisphosphonate treatment reduce the risk of future cancer? Chin Clin Oncol 2019;8(Suppl 1):S3. doi: 10.21037/ cco.2018.10.08
12. Xie F, Li P, Gong J, et al. The bisphosphonate zoledronic acid effectively targets lung cancer cells by inhibition of protein prenylation. Biochem Biophys Res Commun 2015;467:664-9.

13. Evans KD, Oberbauer AM. Alendronate inhibits VEGF expression in growth plate chondrocytes by acting on the mevalonate pathway. Open Orthop J 2009;3:83-8.

14. Di Salvatore M, Orlandi A, Bagalà C, et al. Anti-tumour and anti-angiogenetic effects of zoledronic acid on human non-small-cell lung cancer cell line. Cell Prolif 2011;44:139-46.

15. Ishtiaq S, Edwards S, Sankaralingam A, et al. The effect of nitrogen containing bisphosphonates, zoledronate and alendronate, on the production of pro-angiogenic factors by osteoblastic cells. Cytokine 2015;71:154-60.

16. Yuen T, Stachnik A, Iqbal J et al. Bisphosphonates inactive human EGFRs to exert antitumor actions. Proc Natl Acad Sci USA 2014;111:17989-94.

17. Meraviglia S, Eberl M, Vermijlen D et al. In vivo manipulation of $\mathrm{V} \gamma 9 \mathrm{~V} \delta 2 \mathrm{~T}$ cells with zoledronate and lowdose interleukin-2 for immunotherapy of advanced breast cancer patients. Clin Exp Immunol 2010;161:290-7.

18. Roelofs AJ, Coxon FP, Ebetino FH, et al. Fluorescent risedronate analogues reveal bisphosphonate uptake by bone marrow monocytes and localization around osteocytes in vivo. J Bone Miner Res 2010;25:606-16.

19. Kothawala P, Badamgarav E, Ryu S, et al. Systematic review and meta-analysis of real-world adherence to drug therapy for osteoporosis. Mayo Clin Proc 2007;82:1493-501.

20. Hansen C, Pedersen BD, Konradsen H, et al. Antiosteoporotic therapy in Denmark - predictors and demographics of poor refill compliance and poor persistence. Osteoporos Int 2013;24:2079-97.

21. Stachnik A, Yuen T, Iqbal J, et al. Repurposing of bisphosphonates for the prevention and therapy of nonsmall cell lung and breast cancer. Proc Natl Acad Sci USA 2014;111:17995-8000.

22. Davies JT, Delfino SF, Feinberg CE, et al. Current and Emerging Uses of Statins in Clinical Therapeutics: A Review. Lipid Insights 2016;9:13-29. 